\title{
Feinstaub und Lärm machen Herz und Lunge krank
}

\begin{abstract}
Bisher wenig beachtete Ursachen von Herz- und Lungenerkrankungen waren ein Hauptthema der diesjährigen Herz Lungen Messe in Hannover. Sowohl externe Faktoren wie Feinstaub und Lärm als auch die Zusammensetzung der körpereigenen Darmflora haben erheblichen Einfluss auf das Immunsystem.
\end{abstract}

Nach eher konservativer Schätzung der Welt Gesundheits Organisation (WHO) gehen in Westeuropa rund eine Million gesunde Lebensjahre durch Lärm verloren. Nach einer Faustregel entspricht jede Verdopplung der subjektiv empfundenen Lautstärke einer Zunahme von zehn Dezibel, wobei in Umfragen Verkehrslärm an erster Stelle der Lärmbelästigungen genannt wurde, gefolgt von Fluglärm. In der Nähe einer Autobahn ist es etwa 80 Dezibel laut. Direkte Hörschäden durch Lärm können ab etwa 90 Dezibel auftreten, aber auch geringere Lärmpegel können dauerstressen und dadurch krank machen. Wie Prof. Thomas Münzel, Universitätsmedizin Mainz, berichtete, hängt es aber auch

\section{Darm reguliert das Immunsystem}

Ebenfalls im Zusammenhang mit einer Reihe von kardiologischen und pulmonalen Erkrankungen (insbesondere Asthma) scheint ein interner Faktor zu stehen, dessen zentrale Rolle für die Immunabwehr lange unterschätzt wurde: Das Darmmikrobiom mit seinen rund 10.000 Bakterienarten zuzüglich großer Mengen weiterer Mikroorganismen. Im Körper sind fast zehnmal so viele Bakterien wie Körperzellen, wie Prof. Hortense Slevogt vom Universitätsklinikum Jena erläuterte. Das Mikrobiom enthält hundertmal mehr genetische Informationen als das menschliche Erbgut. Es ist im Alter von drei Jahren vollentwickelt und wirkt prägend auf das Immunsystem sowie die Ausbildung von Toleranzen gegen $\mathrm{Mi}$ kroorganismen. Wie Slevogt erklärte, konnte im Mausmodell gezeigt werden, dass zum Darmmikrobiom gehörende LactobacillenSpezies die regulatorische T-Zell-Antwort

von subjektiven Einschätzungen ab, welche Lautstärken als inakzeptabel empfunden werden: So wird Flugzeuglärm heute als nerviger eingestuft als noch vor 30 Jahren, wohingegen der Verkehrslärm heute eher toleriert wird als früher. Tatsächlich konnte in Studien gezeigt werden, dass insbesondere Fluglärm Ärger auslöst und in der Folge depressiv und ängstlich machen kann. Beides - Flug- und Straßenlärm - ist mit einem signifikant erhöhten Risiko für Bluthochdruck assoziiert.

Nächtliche Überflüge sorgen selbst im Schlaf für Blutdruckspitzen, weshalb die Autoren einer als „Heathrow-Studie“ (Hansell AL et.al. BMJ 2013;347:f5432) bezeichneten epidemiologischen Erhebung in der Lärmbelästigung einen neuen kardiovaskulären Risikofaktor sehen. Inwieweit sich die Gesundheitsgefahren aus Verkehrslärm und Feinstaub addieren, bleibt derweil Gegenstand weiterer Untersuchungen, wobei weder Lärm noch Staub in gängigen kardiologischen bzw. pneumologischen Leitlinien als Risikofaktoren vertreten sind. Dabei sind die nachteiligen Auswirkungen der Luftverschmutzung auf Herz und Lunge hinlänglich bekannt. Verschmutzte Luft ist laut WHO der Umweltfaktor mit dem schädlichsten Einfluss auf die Gesundheit. Die WHO beziffert die Zahl vorzeitiger Todesfälle durch schmutzige Luft auf weltweit sieben Millionen Menschen. modifizieren und vor Asthma schützen können. Umgekehrt könne eine Dysbalance der mikrobiellen Kolonisation pulmonale allergische Reaktionen triggern. Zudem reagierten Mäuse mit ballaststoffreicher Ernährung nach Allergenexposition mit einer verminderten allergisch-inflammatorischen Immunantwort der Lunge im Vergleich zu ballaststoffarm ernährten Tieren.

\section{Gibt es ein Lungenmikrobiom?}

Bis vor etwa zehn Jahren galt die Lehrmeinung, dass die unteren Atemwege steril sind. Heute wird jedoch davon ausgegangen, dass sich Kleinstlebewesen durch Mikroaspiration, Inhalation und Immigration dauerhaft in der Lunge ansiedeln und ein eigenes Lungenmikrobiom bilden, insbesondere dann, wenn die mukoziliäre Clearance gestört ist (Bassis CM et al. MBio 2015 ;6(2):e00037).

Ein besseres Verständnis der Zusammenhänge zwischen den verschiedenen Mikrobiomen und der Entstehung von Krankheiten könnte zur Entwicklung spezifisch darauf abgestimmter Therapien (wie Antibiotika, Probiotika, fäkale Transplantation) führen, so Slevogt.

Dr. Beate Grübler

Quelle: Hannover Herz Lungen Messe, 3. - 5. Juni 2016 